\title{
A LEI DOS EFEITOS ACELERADOS DA ENTROPIA E O LIMITE NO USO DE RECURSOS ENERGÉTICOS DA BIOSFERA
}

\author{
Roberto T. Hosokawa \\ Edson G. Hosokawa
}

\begin{abstract}
RESUMO
O presente texto pretende demonstrar o comportamento da lei dos efeitos acelerados da entropia provocado pela economia antrópica, enfocando principalmente o uso indiscriminado dos recursos energéticos da biosfera e as suas consequiências para a espécie humana.

Palavras chaves: anatomia dos efeitos da entropia natural, quanta anamórficas no universo, economia antrópica, biodiversidade, gradiente do espectro da vida.
\end{abstract}

\section{THE LAW OF THE ENTROPY ACCELERATED EFFECTS AND THE LIMIT IN THE USE OF THE BIOSPHERE ENERGETICS RESOURCES.}

\begin{abstract}
The present text intends to demonstrate the behavior of the law of the entropy accelerated effects provoked by the human economy, foccusing the indiscriminated use of the biosphere energetics resources and its consequences mainly for the human species.
\end{abstract}

Key words: anatomy of the natural entropy. effects, anamorphics quanta in the universe, human economy, biodiversity, spectrum of the life.

\section{INTRODUÇÃO}

Desde o momento em que o ser humano descobriu o fogo como uma forma de energia, a alavanca para mover grandes massas e a roda para transportar as mesmas em longas distâncias, iniciou-se, então, a produção significativa de entropia e que foi potencializada geometricamente com o advento da era industrial, gerando assim a forma mais nefasta de poluição até então conhecida.

A pressão exercida pela população no uso de recursos energéticos, já apresenta efeitos da entropia em escala nos diversos sentidos, ensejando assim a necessidade de se encontrar rumos mais equilibrados na formulação e implementação dos planos de desenvolvimento .

Os objetivos básicos do presente trabalho consistem em provar a teoria que rege a lei dos efeitos da entropia natural e também analisar as conseqüências, da aceleração dos efeitos da entropia na biosfera, provocadas pela economia antrópica.

\section{A Entropia}

A entropia é definida na física como "quantidade de energia de um sistema que não pode ser convertida em trabalho mecânico sem comunicação de calor a algum outro corpo , ou aumento de volume e cresce em todos os processos irreversíveis , ficando constante nos reversíveis."

No meio biológico, a entropia pode ser interpretada como oriunda da desintegração da matéria (alimento) e conseqüente produção de calor e trabalho. É o que ocorre na cadeia alimentar dos seres vivos , através da transferência de calorias.

Além destas características, ela se manifesta com forma definida de evolução acompanhando o consumo de energia . GABOR et al. (1976) contabilizaram a produção e consumo de energia em

1 , hosokawa@ floresta.ufpr.br, pós-doutorado,pesquisador bolsista do CNPq, Professor Sênior da UFPR., 80210-170 - Rua Lothário Meissner, 3400-Curitiba-PR-BR

${ }_{2}^{2}$ Acadêmico de Engenharia Elétrica da UFPR 
função de tempo e chegaram a conclusão de que, no período considerado , cresceram exponencialmente. Como os mesmos são processos irreversíveis,

(a energia não é reciclável), a entropia também cresceu exponencialmente. MEADOWS (1972) explicita que a poluição ambiental cresce exponencialmente, a produção de clorofluormetano (CFM), com efeito, marcante na redução da camada de ozônio (JUNGE, 1975; GUESTEN, 1976; FLOHN, 1977) e a produção de dióxido de carbono (MEADOWS, 1972) são alguns dos processos estudados que mostram crescimento exponencial. Como ocorre em todos esses casos a comunicação de calor, a entropia também cresce exponencialmente.

\section{DESENVOLVIMENTO DO TRABALHO}

Para o desenvolvimento do trabalho foram estudados os seguintes aspectos:

\section{Anatomia dos Efeitos da Entropia Natural (formulação teórica)}

Como todos os processos de evolução da entropia até agora estudados cresceram em forma exponencial positiva, consequentemente os seus efeitos deverão se manifestar de forma exponencial negativa.

Além do mais um conjunto de teorias explicam a sua anatomia:

Pela Teoria de Relatividade Geral de Einstein : a geometria da dinâmica dos eventos no Universo é uma hélice cilíndrica, fig. 1.

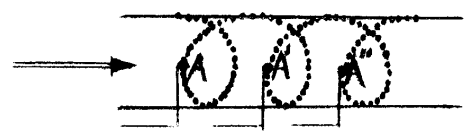

Fig. 1 Dinâmica de Eventos.

A Teoria de Hawking complementa o assunto expressando que eventos cíclicos naturais pertencem ao mesmo cone de eventos, fig. 2.

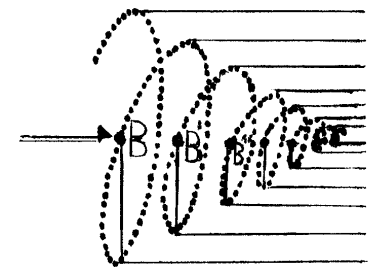

Fig. 2 Cone de Eventos.

Levando em consideração a Teoria de Gravitação Universal, a expansão ou a retração dos eventos cíclicos naturais se processam em forma de espiral logarítmica, fig. 3 .

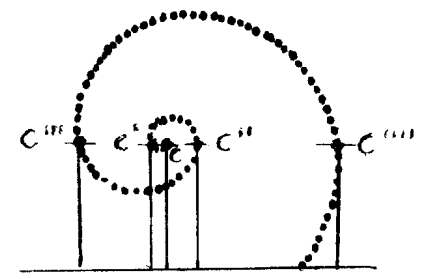

Fig. 3 Gravitação Universal.

O que permite deduzir que os efeitos da entropia natural são eventos cíclicos naturais cuja anatomia é uma hélice cônica, espiralada logarítmica em retração e a sua representação algébrica simplificada é uma função exponencial negativa da dinâmica temporal e espacial do calor, fig. 4.

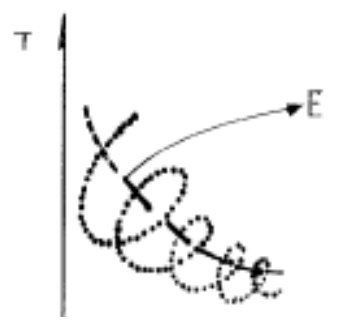


Fig. 4. Efeitos da Entropia Natural natural.

Para a resolução matemática desta função são necessárias as taxas dos efeitos da entropia

Partindo da afirmativa de que a quantidade total de energia no Universo é constante, (Freedman citado por OLIVEIRA F. e SARAIVA 2000), pode-se então formular a seguinte equação:

$$
\mathbf{Q}=\text { Qer + Qef + Qen + Qea +... Qx }
$$

onde:

Q : quantidade total de energia no Universo

Qer: quantidade de energia represada

Qef: quantidade de energia em fluxo

Qen: quantidade de entropia natural

Qea: quantidade de entropia provocada pelo homem

Qx: componente aleatório.

Considerando agora, apenas a energia em fluxo e a sua entropia natural, os efeitos da mesma podem ser analisados pelo consumo da energia numa escala de espaço-tempo (xt).

Seja Qefi a quantidade de energia inicial e qi a energia consumida num determinado intervalo de espaço-tempo. Então é possível calcular as taxas dos efeitos da entropia (Ei) em sucessivos intervalos de espaço-tempo $(\mathrm{xt})$.

$$
\text { E1 }=(\text { Qefi }- \text { qi) }) / \text { Qefi } ; \text { E2 }=(\text { Qef1 }- \text { q2) } / \text { Qef1 } ; . . . ; \text { En }=(\text { Qefn-1 }- \text { qn }) / \text { Qefn-1 }
$$
estatístico :

Se estas taxas forem constantes, então pode ser representado por um modelo analógico-

$$
\mathrm{E}=1 * \mathrm{e}^{-\mathbf{a x t}}+\boldsymbol{\&}
$$

Onde :

E : efeitos da entropia natural; $\quad 1$ : coeficiente linear;

e: número natural;

a: Coeficiente angular;

xt : espaço-tempo;

\& : componente aleatório

\section{Quanta Anamórficas no Universo}

Logaritmizando a função (3), obtém-se:

$$
\ln E=\ln 1+(- \text { axt }) \ln e
$$

e fazendo:

$$
\ln \mathrm{E}=\mathrm{Y} ; \ln \mathrm{I}=\mathrm{L} ;-\mathrm{a}=-\mathrm{A} ; \mathbf{x t}=\mathrm{X} \text {; }
$$

resulta no modelo linear

$$
\mathbf{Y}=\mathbf{L}-\mathbf{A X}
$$

onde:

L : coeficiente linear

A : coeficiente angular 
Variando o valor de L, pode-se obter uma família de curvas anamórficas dos efeitos da entropia natural e, consequentemente, pode-se conceituar o fluxo da estrutura energética no Universo como "quanta anamórficas" que se inicia a altas temperaturas (mais infinito) e através da taxa de entropia natural tende a um valor próximo de zero, quando o espaço tempo tende a (mais infinito)

O coeficiente angular representa a velocidade dos efeitos da entropia natural, que no caso é uma constante. A sua alteração significa em imprimir aceleração, ou seja ,passa a ter um trajeto polimórfico, destoando com a tendência harmonizada anamórfica do Universo.

\section{Efeitos Acelerados da Entropia Provocados pela Economia Antrópica}

De acordo com o descrito anteriormente, os efeitos da entropia serão acelerados quando se altera o coeficiente angular.

A título de consolidação da teoria, analisa-se a seguir o processo da dinâmica de diminuição progressiva da área da fitomassa e a da massa de Araucaria angustifolia provocado pela ação humana no Estado de Paraná, sendo que a grande parte da mesma foi consumida energeticamente (queima).

Utilizando os dados percentuais transformados do INPE (SOS Mata Atlântica) e de (Maack citado por HOSOKAWA 1976) sobre a diminuição das áreas da fitomassa no Paraná, e procedendo a sua análise estatística, chegam-se às seguintes equações dos efeitos da entropia natural e dos da entropia acelerada pela economia antrópica.

Desde o descobrimento do Brasil (1500) até o ano de 1912, a percentagem da área da fitomassa total era de $84,72 \%$, sendo que a partir de 1912 é representada pela equação :

$$
\mathrm{Et}=1365186 * \mathrm{e} \quad-\mathbf{0 , 0 2 3 3 7 5 * t}
$$

sendo:

$$
\mathrm{R}^{2}=94,04 \% \text { e S yx } \%=17,01 \% .
$$

Para a área da massa de Araucaria do ano 1500 até 1930 a percentagem era de 41,50\% e a partir de 1930 é representada pela equação:

$$
\mathrm{Ea}=2270693 * \mathrm{e}
$$$$
-0,026733152 * t
$$

sendo:

$$
\mathrm{R}^{2}=96,66 \% \text { e Syx } \%=9,63 \%
$$

Os efeitos da entropia natural são representados pelas seguintes equações:

\section{$\mathbf{I}-\mathbf{0 I} * \mathbf{T}$}

$$
\text { Ent }=84,72 * \mathrm{e}
$$

$\mathrm{e}$

\section{$\mathbf{I - 0 I} * \mathbf{T}$}

$$
\text { Ena }=41,5 * \mathrm{e}
$$

São funções que representam valores constantes na escala de tempo absoluto.

Os efeitos da ação antrópica são descritos, respectivamente, pelos seguintes resultados: 
Para a área de fitomassa total:

$$
\begin{array}{cc}
\mathbf{I - 0 I} * \mathbf{T} & \mathbf{- 0 , 0 2 3 3 7 5} * \mathbf{T} \\
\text { Ent }=\text { Et }=84,7 / 2 * \mathrm{e} & =1365186 * \mathrm{e}
\end{array}
$$

e para a da Araucaria angustifolia:

$$
\begin{aligned}
& \mathrm{I}-0 \mathrm{I} * \mathrm{~T} \quad \mathbf{- 0 , 0 2 6 7 3 3 1 5 2} * \mathrm{~T} \\
& \text { Ena }-\mathrm{Ea}=41,5 * \mathrm{e}-2270693 * \mathrm{e}
\end{aligned}
$$

A modelagem matemática-estatística dos dados demonstra claramente os efeitos acelerados da entropia provocados pela economia antrópica. Em termos de escala cósmica, o tempo decorrido desde o descobrimento do Brasil até o presente ano, é apenas um lapso de um momento extremamente fugaz. Contudo o homem teve a proeza de destruir a fitomassa de Araucaria angustifolia em pouco mais de 70 anos, sendo que a longevidade natural da espécie pode atingir séculos.

\section{Conseqüências da Alteração nos Efeitos da Entropia na Biosfera}

Levando em consideração a dinâmica da estrutura energética (quanta) numa escala de espaçotempo como um ícone anamórfico para as galáxias, então todo o Universo é composto de elementos e seres que nada mais são do que uma determinada anatomia energética em um dado momento. Se os componentes do Universo estão sujeitos a transformações na sua estrutura energética de uma forma harmônica ao longo do espaço-tempo, é de se esperar que seres vivos, cuja estrutura energética divergir significativamente dos mesmos, tenderão à extinção.

Isto sugere a relação equilibrada existente entre a estrutura energética do Universo com a biodiversidade e o espectro da vida.

\section{A Inserção da Biodiversidade em Quanta Anamórficas do Universo}

A figura 5 visualiza a relação entre a biodiversidade e a energia no contexto da dinâmica da temperatura em função de espaço-tempo.

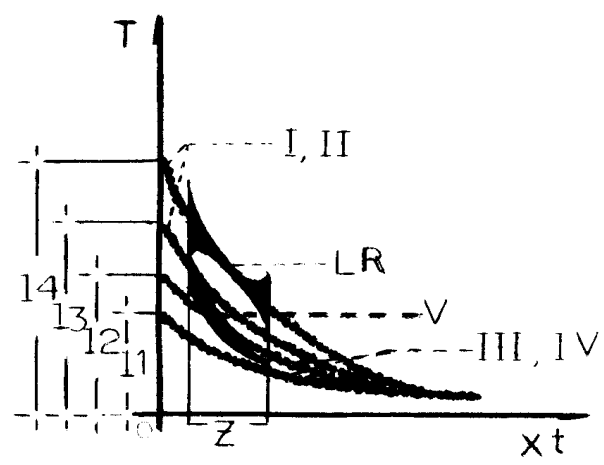

Fig. 5 . Coeficientes da Biodiversidade.

As curvas I,II,III e IV, representam os efeitos da entropia natural da biosfera em harmonia com os do Universo. 
A curva $\mathrm{V}$ mostra o efeito acelerado da entropia provocado pela economia antrópica (faixa $\mathrm{Z}$ é o período da existência do homem até os dias de hoje)

As curvas de I a IV, por exemplo, representam a evolução dos diversos níveis de estrutura energética e caracterizam diferentes ambientes que permitem abrigar a diversidade da vida dentro dos seus respectivos limites da resiliência biológica (LR). Neste contexto , pode-se afirmar que, cada curva caracteriza um ambiente que permite a existência de um conjunto de seres vivos e, portanto pode ser aferido um coeficiente de biodiversidade $(11 ; 12 ; 13 ; 14)$.

A alteração significativa do meio ambiente, desorganizando-o energeticamente com processos de alta entropia , produz aceleração nos efeitos da mesma (curva V), conduzindo o conjunto de vidas a um outro ambiente energético e consequentemente passam a sofrer demandas nas alterações genéticas.

As espécies que conseguirem se adaptar com evoluções genéticas sobreviverão. Caso contrário serão espécies em extinção.

\section{Gradiente do Espectro da Vida.}

Logaritmizando a equação dos efeitos da entropia natural, chega-se a uma função linear (5) e fig. 6, que quando aplicado a um determinado meio de biodiversidade, obtém-se diversos coeficientes de espécies, pois variando o coeficiente linear (L) caracteriza-se um gradiente do espectro de vida. (cada espectro é um ambiente energético específico para cada espécie).

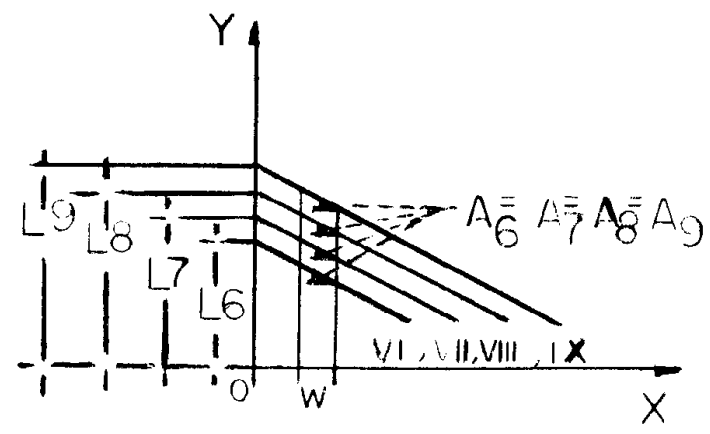

Fig. 6. Coeficientes das Espécies.

Isto explica, porque no mesmo tempo e no mesmo espaço, podem existir diferentes espécies caracterizadas por estruturas energéticas específicas compatíveis com o meio de cada uma.

A faixa w representa o gradiente energético natural em um dado momento, pois as curvas VI a IX possuem o mesmo coeficiente angular (A6; A7; A8; A9), mas com diferentes

níveis energéticos definidos pelos coeficientes lineares (L6; L7; L8; L9).

Por isso existem no mesmo tempo e espaço (biosfera) seres que vivem próximos das lavas vulcânicas e outras na gélida Antártida.

\section{CONCLUSÕES}

A principal conseqüência em relação ao homem seria não possibilitar tempo necessário para sua adaptação somática em relação às alterações do meio ambiente, evocando assim maiores riscos de extinção da própria espécie.

A prudência indica que deve se utilizar, na medida do possível, processos cujos efeitos da entropia estejam equilibrados com os da biosfera. Em outros termos, seria utilizar energia em fluxo como: energia de hidroelétricas, energia solar, energia eólica, fitomassa em produção sustentada (fotossíntese), energia dos fluxos de mar, arco foto-voltaico, e minimizar o uso de energias concentradas como carvão de pedra, petróleo, urânio. 
É necessário desenvolver tecnologias que produzem efeitos de entropia compatíveis com o meio ambiente.

Em termos de desenvolvimento sustentável, o processo adotado deve ser compatível com sustentabilidade relativa entre o microcosmo e o macrocosmo, já que a absoluta não existe no Universo.(HOSOKAWA, 2000)

Não alterar a estrutura energética do meio a ponto de extrapolar os limites da resiliencia biológica das espécies que nele vivem. Neste sentido o homem já alterou significativamente o meio em que vive, tornando-se cada vez mais dependente de recursos exosomáticos como aquecimentos para superar o frio, antibióticos para combater as enfermidades, tecnologias para transportes de massas baseadas em processos de alta entropia .

\section{BIBLIOGRAFIA CITADA}

FLOHN, H. (1977): Stephen wir vor einer Klima Katastrophe? Umschau in Wissenschaft und Technik, H. 17.

GABOR, D.; COLOMBO, U. A. (1976): Das Ende der Verschwendung zur materiellen Lage der Menschheit - Ein Tatsachenbericht an den Club of Rome, Deutsche Verlag Anstalt. Stuttgart.

GUESTEN, H. (1976): So durchlöchen wir die Atmosphaere. Bild der Wissenschaft. 3.

HOSOKAWA, R.T. (1976): Betriebswirtschaftliche Kriterien zur Wahl der Umtriebszeit von Araucaria angustifolia (BERT. / O. KUNTZE) in Brasilien, Tese de Doutorado, Universidade Albert Ludwig de Freiburg, Alemanha, 255 pgs.

HOSOKAWA, R.T.(2000): O Paradoxo no Desenvolvimento Sustentável, SBPN Scientific Journal, Curitiba, v.4, Suplemento 1, pg. 5-8, .

JUNGE, Chr. (1975): Das Kohlendioxid und seine Zunahme, Promet meteorologische Fortbildung, H. 2.

MEADOWS, D. (1972): Die Grenzen des Wachstums - Bericht der Club of Rome zur Lage der Menschheit. Deutsche Verlag Anstalt, Stuttgart.

OLIVEIRA Fo., K. de S.; SARAIVA, M. de F. O. (2000): Astronomia e Astrofísica, Porto Alegre, UFRGS, $585 \mathrm{p}$. 